\title{
Quasiparticle Dynamics in the Kondo Lattice Model at Half Filling
}

\author{
Simon Trebst ${ }^{1,2}$, Hartmut Monien ${ }^{3}$, Axel Grzesik ${ }^{3}$, and Manfred Sigrist ${ }^{1}$ \\ ${ }^{1}$ Theoretische Physik, Eidgenössische Technische Hochschule Zürich, CH-8093 Zurich, Switzerland \\ ${ }^{2}$ Computational Laboratory, Eidgenössische Technische Hochschule Zürich, CH-8092 Zurich, Switzerland \\ ${ }^{3}$ Physikalisches Institut, Universität Bonn, Nußallee 12, 53115 Bonn, Germany
}

(Dated: December 23, 2018)

\begin{abstract}
We study spectral properties of quasiparticles in the Kondo lattice model in one and two dimensions including the coherent quasiparticle dispersions, their spectral weights and the full twoquasiparticle spectrum using a cluster expansion scheme. We investigate the evolution of the quasiparticle band as antiferromagnetic correlations are enhanced towards the RKKY limit of the model. In both the $1 \mathrm{D}$ and the $2 \mathrm{D}$ model we find that a repulsive interaction between quasiparticles results in a distinct antibound state above the two-quasiparticle continuum. The repulsive interaction is correlated with the emerging antiferromagnetic correlations and can therefore be associated with spin fluctuations. On the square lattice, the antibound state has an extended s-wave symmetry.
\end{abstract}

PACS numbers: 71.27.+a, 71.10.Fd

The Kondo lattice is one of the fundamental microscopic models for the description of heavy fermion materials. The basic ingredients are nearly localized $f$ electrons on every lattice site and itinerant conduction electrons which are non-interacting apart from a local coupling to the $f$-electrons. In the Kondo lattice model the $f$-electrons form localized spin degrees of freedom and the coupling is represented as an onsite exchange interaction between this spin and the conduction electron spin density. This rather simple model gives rise to complex many body physics whose detailed understanding is still far from complete. Generally it is believed, that many of the properties of heavy Fermion systems originate from the interplay between magnetic RKKY interaction among the localized spins and the Kondo effect screening these spins ${ }^{1.2 .3}$. The former leads to a longrange ordered antiferromagnetic phase in two and three dimensions and the latter to a phase with short-ranged spin correlations due to the formation of coherent Kondo spin singlets. There is a quantum phase transition between the two limiting phases upon changing the parameters of the model ${ }^{1.2 .3}$.

For the metallic heavy Fermion systems 2.4 this quantum phase transition has recently become one of the highpriority issues. In particular, the behavior of the charge carriers at the quantum phase transition has gained special interest and questions have been raised on the character of the Fermi surface, whether it is "large" or "small" 3 . In the case of a large Fermi surface both the conduction electrons and the $f$-electrons forming the localized spins are included in the Fermi volume. The small Fermi surface, in contrast, consists only of the conduction electrons. It seems obvious that in the case of magnetic long-range order the $f$-electrons tend to loose their mobility and drop out in the Fermi volume count. On the other hand, the generally accepted picture of the heavy Fermion phase is that the electrons at the Fermi level have strong $f$-character such that the $f$-electrons are in the Fermi volume. Consequently a swift change of the Fermi surface topology is expected at the magnetic quan- tum phase transition ${ }^{2}$. In a recent experiment Paschen et al. have indeed observed a characteristic feature in the evolution of the Hall effect of $\mathrm{YbRh}_{2} \mathrm{Si}_{2}$ through the quantum phase transition, consistent with this picture ${ }^{5}$.

The Kondo insulator which corresponds to the halffilled Kondo lattice with one conduction electron per localized spin is a special phase where the quantum phase transition can be more easily discussed, since there are no low-lying quasiparticle degrees of freedom. In the socalled strong coupling regime the Kondo effect dominates yielding a spin liquid phase with a spin and a charge gap in the excitation spectrum. On the other hand the antiferromagnetically ordered phase in the weak coupling regime has only a charge gap while the spin sector possesses gapless spin wave modes.

While under these circumstances the issue of the Fermi surfaces is not of immediate relevance, examining the behavior of the quasiparticle and charge excitations will still give much insight into the fate of the quasiparticle spectrum for the system in the vicinity of the quantum phase transition. In particular, the approach from the quantum disordered side allows us to investigate the modification of the quasiparticle spectrum which reflects the gradually extending magnetic correlations in the approach of the quantum phase transition. It is also worth to analyze how the enhancement of spin fluctuations affects the two particle excitations. One of the important questions is the spin fluctuation mediated interaction between two particles, whether they can form bound pairs. This would give insight into the possibility of unconventional superconductivity in weakly doped Kondo lattice systems.

Some of these issues have been addressed recently by Assaad and coworkers based on Quantum Monte Carlo methods as we will discuss later ${ }^{6.7 .8}$. Discussion of the behavior of the half-filled Kondo lattice model and the related periodic Anderson model in a magnetic field examined the behavior of the quasiparticle spectrum at quantum phase transitions from a different view point ${ }^{9.10}$. In these latter studies the quantum phase transition to magnetic long-range order has been induced by a mag- 
netic field which lowers the spin triplet excitations and the modifications of the quasiparticle spectrum discussed. Both studies show that the system always remains in an insulating phase $\frac{9.10}{.}$.

The Hamiltonian of the Kondo lattice model (KLM) is given by

$$
H_{K L M}=-t \sum_{\langle i j\rangle, \sigma}\left(c_{i \sigma}^{\dagger} c_{j \sigma}+\text { h.c. }\right)+J \sum_{i} S_{i} \cdot S_{i}^{c},
$$

where $S_{i}^{c}=\frac{1}{2} \sum_{\sigma, \sigma^{\prime}} \tau_{\sigma, \sigma^{\prime}} c_{i \sigma}^{\dagger} c_{i \sigma^{\prime}}$ are the spin density operators of conduction electrons and $S_{i}$ are localized $f$ spins at site $i$ with $\tau_{\sigma, \sigma^{\prime}}$ being the Pauli matrices. The magnetic exchange coupling $J$ derived from the periodic Anderson model is antiferromagnetic $(J>0)^{11}$. Furthermore, $t$ denotes the hopping-matrix element which we restrict to nearest-neighbor hopping only. The Kondo insulating phase is realized for $t / J \ll 1$ where $t / J$ will be our small expansion parameter.

The strong-coupling limit $(t / J \rightarrow 0)$ represents indeed a good starting point for a well-controlled perturbative approach based on a systematic cluster expansion ${ }^{12}$. This serves well our goal to study of the evolution of the quasiparticle spectrum in view of the emerging longer ranged antiferromagnetic correlations as $t / J$ is gradually increased. In the following sections we will discuss the oneand two-dimensional bipartite Kondo lattice model. Although the former does not have a quantum phase transition, the antiferromagnetic correlations increase strongly for growing $t / J$. In two dimensions, however, a quantum phase transition is expected for $t / J \approx 0.68^{6}$.

\section{THE ONE-DIMENSIONAL MODEL}

\section{A. The quasiparticle spectrum}

We first discuss spectral properties of quasiparticle (hole) excitations for the one-dimensional KLM at halffilling. Starting from the limes of decoupled singlets on the lattice sites for the Kondo insulator $(t / J=0)$ we have calculated high-order strong-coupling expansions in the hopping strength $t / J$. This allows us to block-diagonalize the Hamiltonian by integrating out spin and charge fluctuations up to a given order and calculate effective Hamiltonians for the ground-state at half-filling, and the degenerate manifolds with a single or two quasiparticle (hole) excitations ${ }^{12,13.14}$. Complementary to previous approaches using bond operator techniques ${ }^{7.15}$ our strongcoupling expansion treats charge and spin fluctuations on the same footing without the need of any mean field approximations. Previous numerical work have studied the nature of the ground state of the 1D model as well as the elementary spin, quasiparticle and charge gaps, such as an extensive DMRG study $\stackrel{16}{\underline{16}}$ and strong coupling analysis 17.18 . Here we present results of a strong coupling analysis for the single and two particle properties.

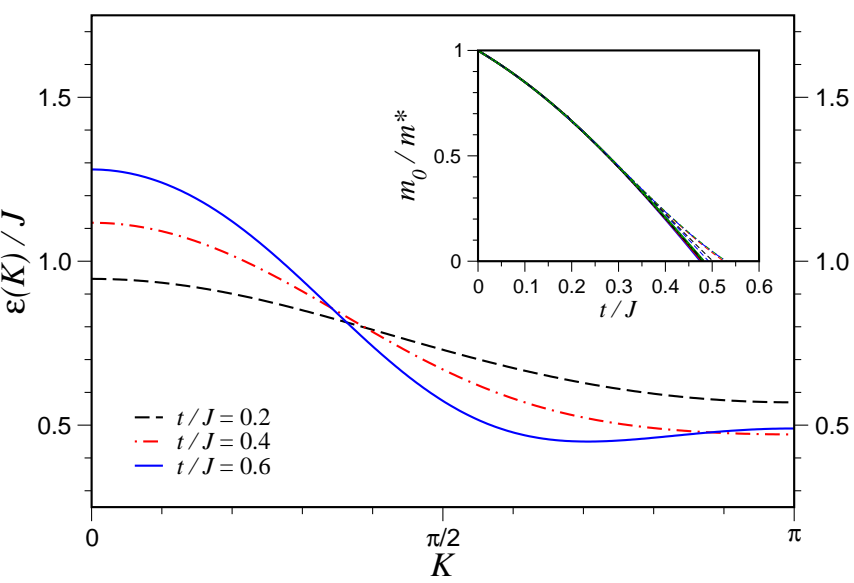

FIG. 1: Dispersion of quasiparticles in the 1D Kondo lattice model for various hopping amplitudes $t / J$ obtained by an 11th order strong coupling expansion around the Kondo insulator. The series were extrapolated using optimized perturbation theory. The inset shows the reciprocal effective mass. Several Padé and Dlog Padé approximant are shown, as well as parabolic fits to the dispersion obtained by optimized perturbation theory.

The dispersion of a single quasiparticle excitation

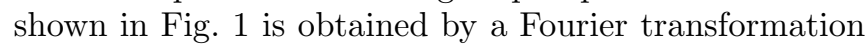
of the respective effective Hamiltonian which we have calculated up to 11 th order in $t / J$. The minimum of the quasiparticle (hole) dispersion is found at momentum $K=\pi$. With increasing hopping strength the band flattens around its minimum exhibiting a continuous enhancement of the effective mass. This effect is connected with the growing "coherence" among the $f$-electrons as the localized spins start to be correlated. This behavior very much resembles what is seen in theories discussing the heavy Fermion physics in the periodic Anderson model in terms of a renormalized hybridization of conduction and $f$-electrons which leads to a minimum of the hole excitations at $K=\pi$ with nearly localized $(f)$ character ${ }^{19.20}$. Interestingly our calculations show even a divergent effective mass at $K=\pi$ at a critical value of $t / J$ beyond which a continuous shift of the band minimum away from $K=\pi$ occurs. In the inset of Fig. 11 we plot the reciprocal effective mass $m_{0} / m^{*}$ which we have computed from the series for the quasiparticle dispersion. For various extrapolation schemes including Padé / Dlog-Padé approximants 21 and optimized perturbation theory $(\mathrm{OPT})^{22}$ the effective mass is found to diverge around $t / J \approx 0.50 \pm 0.02$ independent of the order of the expansion (8th to 11th order). Thus the divergence of the effective mass is obviously not an artifact of the strong coupling expansion. Simultaneously for large hopping strength quasi long range $\mathrm{AF}$ order builds up in the one-dimensional system and the spin gap $\Delta_{S}$ remains finite 16.23 .

The shift of the energy minimum is connected with the appearance of effective hopping processes (obtained through integrating out higher energy configurations) 

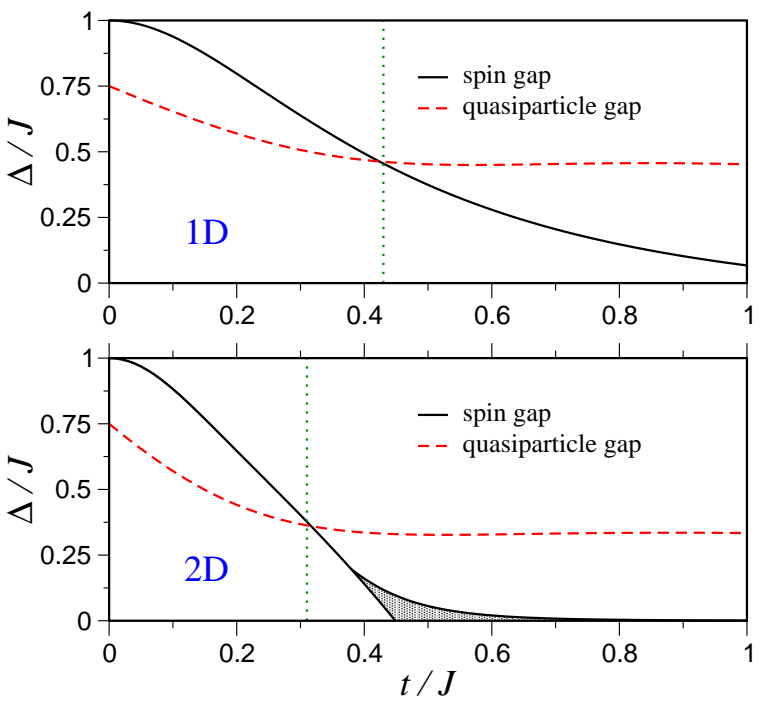

FIG. 2: Spin and quasiparticle gap for the Kondo lattice model. In one dimension (upper panel) the spin gap stays finite for increasing hopping strength. In two dimensions (lower panel) the spin gap vanishes for finite hopping strength, the grey shaded area indicates the variation of the various Padé approximants. The quasiparticle gap $\Delta_{Q P}$ becomes nearly constant for $t / J>0.5$ in both dimensions. In $1 \mathrm{D}(2 \mathrm{D})$ the spin gap crosses the quasiparticle gap $\Delta_{S} \approx \Delta_{Q P}$ around $t / J \approx 0.43(0.36)$ as indicated by the dotted lines.

which are non-bipartite, i.e. connected points on the A (or B) sublattice. To lowest order the dispersion is given by

$$
\varepsilon(K)=t \cos K+\frac{t^{2}}{3 J} \cos 2 K
$$

which yields an effective mass

$$
\frac{m_{0}}{m^{*}}=1+\frac{4 t}{3 J}
$$

with $m_{0}=2 / t$. The second term involves the nextnearest neighbor hopping process. Such hoppings become increasingly facilitated compared to the hopping between different sublattices, as the antiferromagnetic correlation grows. This gives a preference to hole transfers between sites of the same sublattice whose localized spins tend to be parallel. Indeed we observe that the appearance of this behavior is correlated by the decrease of the spin gap below the quasiparticle gap, see Fig. 2 As a consequence, the time scale for spin fluctuations increases and the quasiparticle excitation is dressed by a slowly fluctuating AF spin background.

\section{B. The two-quasiparticle spectrum}

We now turn to the spectrum of two-quasiparticle excitations and explicitly address the question whether two quasiparticles attract or repel each other, thereby forming a distinct bound or antibound state separated from

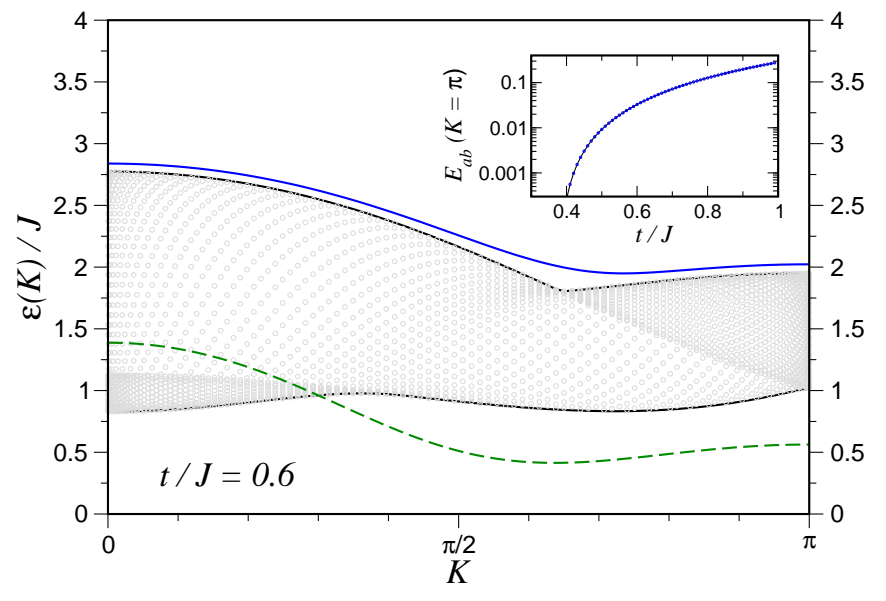

FIG. 3: Spectrum of quasiparticles in the 1D Kondo lattice model. For $t / J>0.4$ an antibound singlet state (solid line) emerges above the continuum (shaded area). The single quasiparticle dispersion is given by the dashed line. The inset shows the antibinding energy of the singlet antibound state at $K=\pi$. Note the logarithmic scale.

the two-quasiparticle scattering continuum. By means of a strong-coupling expansion the two-quasiparticle spectrum is computed by calculating effective Hamiltonians in the two-particle sector integrating out order by order spin and charge fluctuations. The cluster expansion then allows us to determine the exact Schrödinger equation for two quasiparticles in the thermodynamical limit (up to the order calculated) which we then solve numerically 13,14 .

Here we present results from an expansion up to 8th order in the hopping strength $t / J$. The obtained series for the matrix elements of the effective Hamiltonian have been extrapolated by applying the OPT approach ${ }^{22}$. The full spectrum of two quasiparticles is shown in Fig. 3. In addition to the single quasiparticle band (dashed line) there is a continuum of scattering states. While we do not find any bound states, a singlet antibound state emerges on top of the continuum for sufficiently large hopping strength $t / J>0.4$ as illustrated by the solid line in Fig. 3. This antibound state starts to split off from a kink in the upper continuum edge around $K \approx 0.65 \pi$. This kink arises for $t / J>0.45$ as the minimum of the single quasiparticle band starts to wander away from $K=\pi$. The singlet antibound state definitely emerges strongly with the enhanced antiferromagnetic correlations. We find no evidence for a (anti-)bound state in the $S=1$ spin sector.

\section{THE TWO-DIMENSIONAL MODEL}

For the two-dimensional Kondo lattice model antiferromagnetic spin correlation develops with increasing hopping strength much more strongly than in the one-dimensional model and for a finite critical value of 


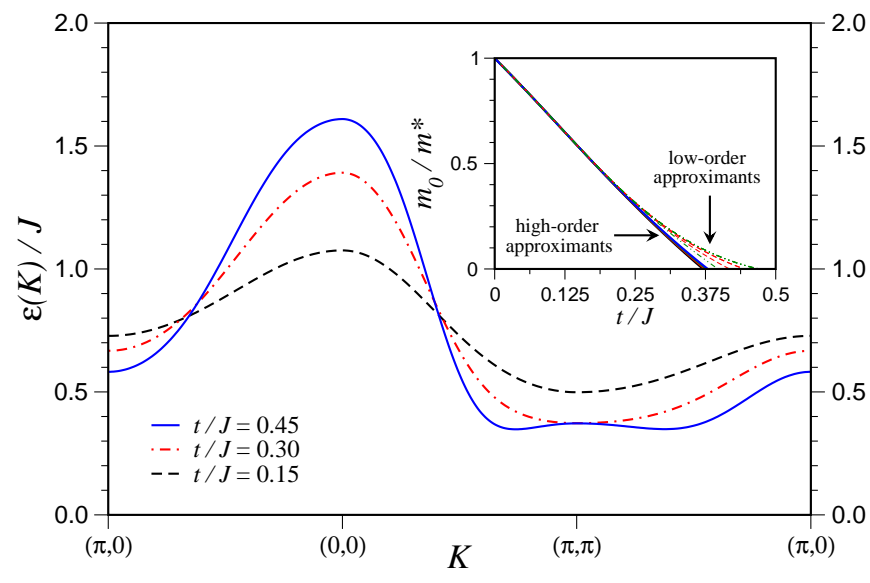

FIG. 4: Dispersion of quasiparticles in the 2D Kondo lattice model for various hopping amplitudes $t / J$ obtained by an 11th order strong coupling expansion around the Kondo insulator. The series were extrapolated using optimized perturbation theory (OPT). The inset shows the reciprocal effective mass. Several Padé and Dlog Padé approximante are shown, as well as parabolic fits to the OPT dispersion.

$(t / J)_{c}$ the system undergoes a quantum phase transition from the Kondo insulator, a phase with gaped charge and spin excitations and short ranged correlations to an antiferromagnetic state with gapless spin excitations and long ranged correlations. The critical point was best determined by a quantum Monte Carlo study ${ }^{6}$ to be $(t / J)_{c}=0.68 \pm 0.02$. Alternative numerical approaches included bond-operator mean field calculations ${ }^{7,15}$ and series expansions ${ }^{17,18}$, which yield similar estimates. Here we present results of a strong coupling analysis for the single and two particle properties.

\section{A. The quasiparticle spectrum}

We first discuss spectral properties of single quasiparticle (hole) excitations at half-filling. The dispersion of the quasiparticle illustrated in Fig. 团has been calculated from a strong-coupling expansion around the Kondo insulator up to 11th order in the hopping strength $t / J$ summing up some 1,691 cluster diagrams. Similar to the onedimensional model, we find the minimum of the dispersion at $K=(\pi, \pi)$. For small $t / J<0.3$ our results agree well with a recent series expansion study ${ }^{18}$ and bond operator mean field calculations ${ }^{7.15}$ which did not take into account spin fluctuations. With increasing hopping amplitude we again observe an increasing band width accompanied by an increase of the effective quasiparticle

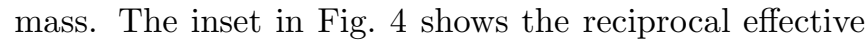
mass $m_{0} / m^{*}$ of the quasiparticle. Analogous to the onedimensional case we encounter the formation of a weakly dispersive part of the band close to $K=(\pi, \pi)$ which also here is consistent with the heavy Fermion picture obtained by means of other methods, but is in contrast

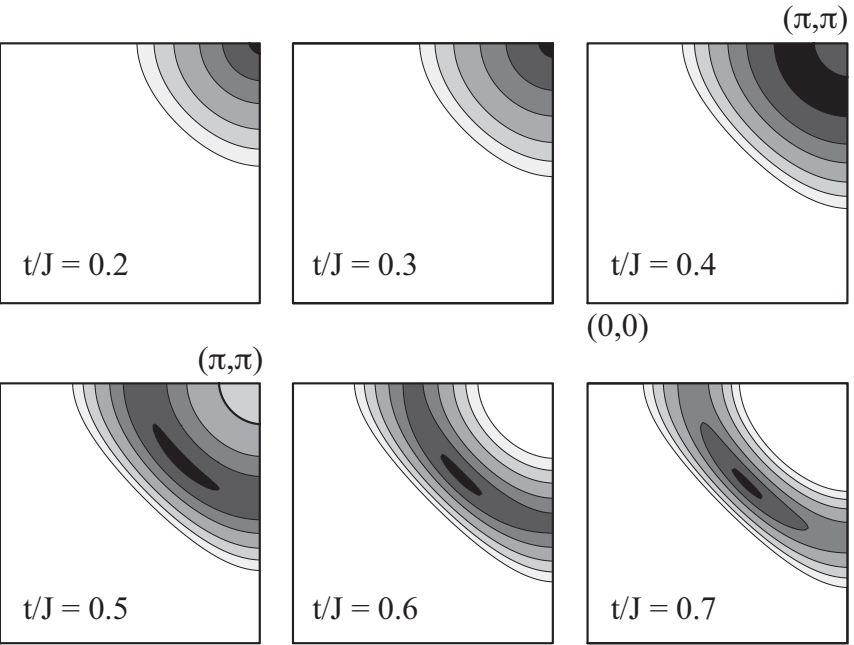

$(0,0)$

FIG. 5: Minimum of the quasiparticle band for the twodimensional Kondo lattice model, where the grey shading/contour lines reflect the distance from the overall minimum (black). The panels describe the path from strong Kondo coupling $(t / J=0.2$, upper left panel) to the antiferromagnetically ordered phase $(t / J=0.7$, lower right panel). The shift of the band minimum towards the zone center at $K=(\pi / 2, \pi / 2)$ for $t / J>0.375$ indicates the onset of antiferromagnetic order before the system undergoes a phase transition at $(t / J)_{c}=0.68$.

to results from a recent quantum Monte Carlo study ${ }^{8}$.

For $t / J \approx 0.40 \pm 0.05$ we find that the effective quasiparticle mass diverges, and the minimum of the quasiparticle band starts to shift toward the zone center at $K=(\pi / 2, \pi / 2)$ as illustrated in Fig. 5 Similar to the one-dimensional model this shift occurs around the hopping strength where the time scales of spin and charge fluctuations become comparable, see the lower panel in Fig. 2. In the same way as for the one-dimensional Kondo lattice we can attribute this behavior to an increased hopping of quasiparticles on the same sublattice. The quasiparticle dispersion thereby reveals the onset of antiferromagnetic spin order well below the transition to the long range ordered state.

\section{B. The two-quasiparticle spectrum}

The singlet antibound state found for the onedimensional model is the result of growing spin fluctuations. In two dimensions the formation of bound or antibound states due to the effective interaction among the quasiparticles has large freedom as we discuss in the Appendix. To study this aspect for the two-dimensional model we have calculated the effective two-quasiparticle Hamiltonian, integrating out spin and charge fluctuations up to 8th order in the hopping strength $t / J$.

Fig. [6 illustrates the full spectrum of quasiparticles in the 2D Kondo lattice model close to the transition 


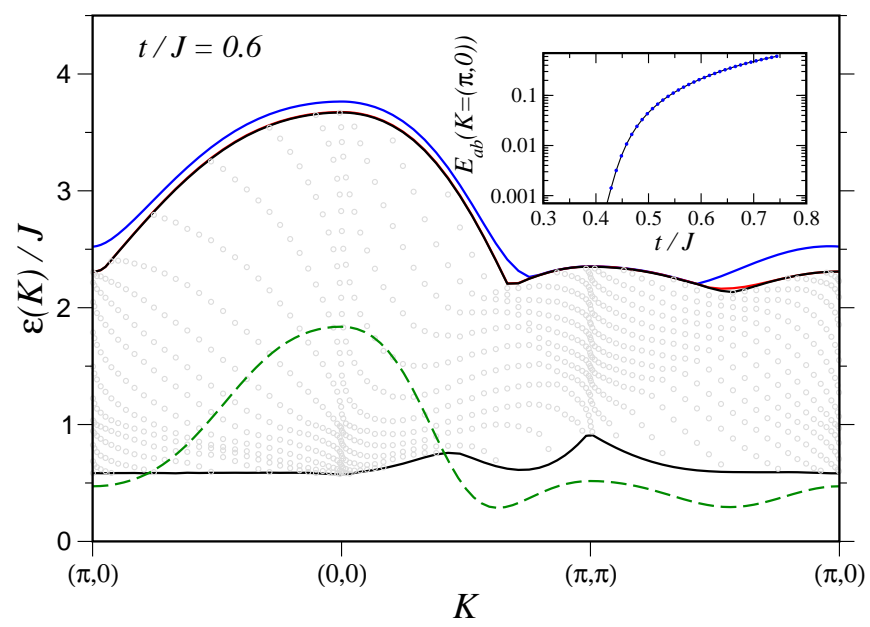

FIG. 6: Spectrum of quasiparticles in the 2D Kondo lattice model. Close to the parameter regime where the effective quasiparticle mass diverges and the time scales of spin and charge fluctuations become comparable, a singlet antibound state with extended $s$-wave symmetry emerges above the continuum. The inset shows the antibinding energy of the singlet antibound state at $K=(\pi, 0)$. Note the logarithmic scale.

to the AF phase. Above the quasiparticle continuum a singlet antibound state (solid line) is found with a dispersion bearing some resemblance to the single quasiparticle band (dashed line). The antibound state only slowly separates from the continuum starting to emerge around $t / J \gtrsim 0.4$. The antibinding energy at $K=(\pi, 0)$ is plotted in the inset of Fig. 6. The circumstance that the singlet antibound state occurs only at finite hopping strength $t / J$ close to the phase transition to the antiferromagnetically ordered state provides further evidence that the repulsive interaction originates from antiferromagnetic spin correlations.

Performing a symmetry analysis as detailed in an Appendix we find that the singlet antibound state has an extended $s$-wave symmetry, since the state can be mapped onto the irreducible representation $A_{1}$ of the $C_{4 v}$ point symmetry group of the two-dimensional square lattice.

\section{CONDUCTION ELECTRONS AND HEAVY QUASIPARTICLES}

We now consider in more detail the quasiparticle spectrum and relate our result with other theories of the heavy Fermion state, which we mentioned already previously. For this purpose we turn to the spectral weight $Z_{Q P}(K)$ of the quasiparticle excitation by expanding the coherent part of the dynamic structure factor

$$
\begin{aligned}
S(q, \omega) & =\int \frac{d t}{2 \pi} e^{-i \omega t} \sum_{r, s} e^{i q r}\left\langle c_{s}^{\dagger}(0,0) c_{s}(r, t)\right\rangle \\
& =Z_{Q P}(K) \delta\left(\omega-\epsilon_{Q P}(K)\right)+\text { incoherent }
\end{aligned}
$$

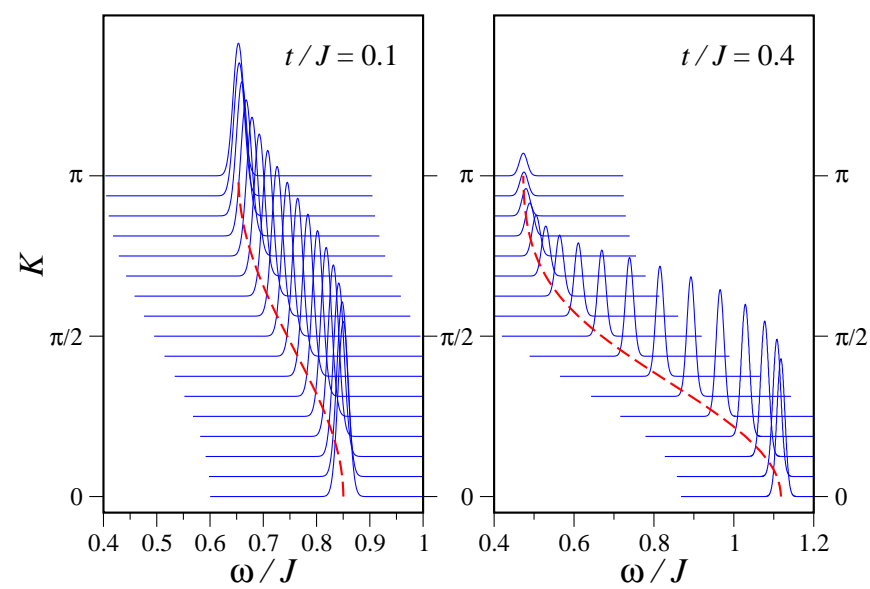

FIG. 7: Spectral weight of a quasiparticle (hole) excitation for the one-dimensional Kondo lattice model. The $\delta$-peaks are broadened by a Gaussian. The dashed lines indicate the dispersion of the quasiparticle. As the hopping amplitude increases a major part of the spectral weight shifts towards the band maximum at $K=0$.

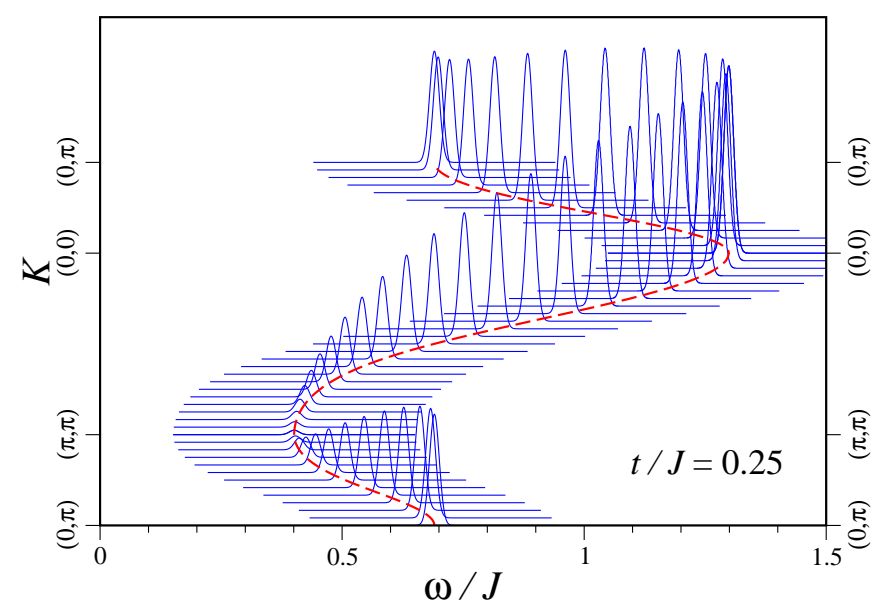

FIG. 8: Spectral weight of a single quasiparticle in the twodimensional Kondo lattice model. The $\delta$-peaks are broadened by a Gaussian. Already for small hopping strength $t / J=$ 0.25 the spectral weight concentrates at the maximum of the quasiparticle band (dashed line) at $K=(0,0)$.

In the strong coupling limit the weight is constant for all $K$. With growing $t / J$ a weight redistribution is observed giving a larger weight on the dispersing part for small $K$ and a pronounced reduction of weight in the nearly flat band region towards $K=\pi$ and $K=(\pi, \pi)$ in one or two dimensions as shown in Figs. 7 and 8 respectively. The

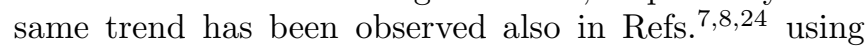
Quantum Monte Carlo techniques. These calculations suggest that the lost weight is absorbed into a "shadow band" being formed due to the enhanced antiferromagnetic correlations. Within the strong coupling expansion the incoherent part is understood as a continuum of the single quasiparticle combined with an independent spin-1 excitation. Out of this continuum the "shadow band" is 


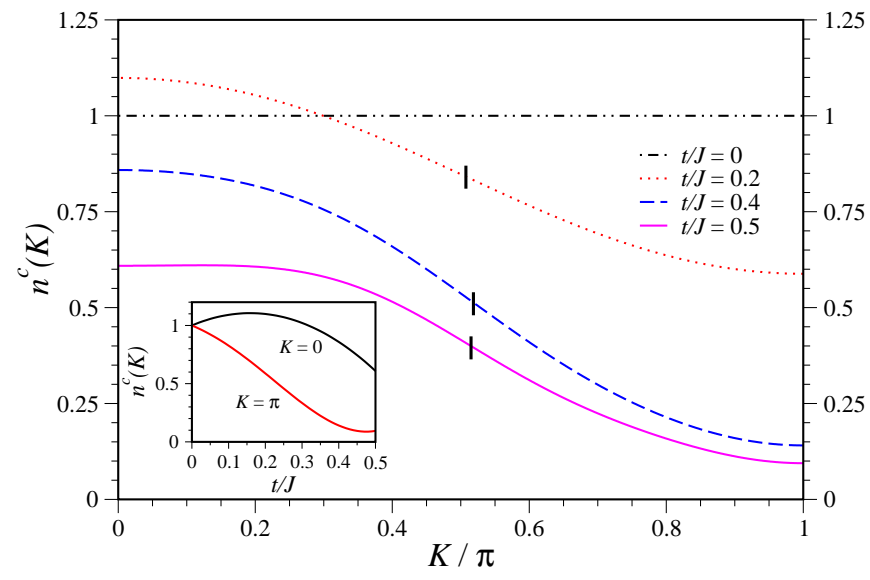

FIG. 9: Integrated weight $n^{c}(K)$ for varying hopping strength $t / J$ in the one-dimensional Kondo lattice model. The inflection points which are marked by the solid bars indicate the position of the conduction electron Fermi surface. The inset shows the particle density at $K=0$ and $K=\pi$ versus the hopping strength.

emerging. We omit a more detailed consideration of the shadow band here and concentrate on the coherent part.

The integrated weight under the coherent quasiparticle peak in $S(q, \omega)$ provides $n_{K}^{c}=\left\langle c_{K s}^{\dagger} c_{K s}\right\rangle$. In Fig. 9 we show $n^{c}(K)$ for the one dimensional system. Obviously the large- $K$ part is depleted of the conduction electron contribution and a relative increase of the density appears in the part of small $K$ where the energy scale is given by the hopping matrix element of the conduction electrons. The same feature occurs in two dimensions as shown in Fig [10] The parts with high weight are bound to eventually become the genuine conduction electron bands. Note that there is an overall drop of quasiparticle weight due to many body effects.

It is interesting to compare our finding with the numerical DMRG results by Ueda and coworkers for the one dimensional Kondo lattice with a finite doping 25 . In their data for $n^{c}(K)$ show a large value for small momenta and a pronounced drop at $K$ corresponding to Fermi momentum of the conduction electrons. For higher momenta $n^{c}(K)$ is much smaller. For finite small doping into this band the real Fermi level lies in the weakly dispersing part, yielding the "heavy" quasiparticles corresponding to a large Fermi surface in the sense of quasiparticle count.

We can also follow the mean field and Gutzwillertype of discussion of the periodic Anderson or CoqblinSchrieffer model which give for half-filling:

$$
n^{c}(K)=\frac{1}{2}\left(1+\frac{\gamma(K)}{\sqrt{\gamma(K)^{2}+\alpha^{2}}}\right)
$$

with $\gamma(K)=2 t \cos K(1 \mathrm{D})$ and $\gamma(K)=2 t\left(\cos K_{x}+\right.$ $\left.\cos K_{y}\right)^{19.20 .26}$. The parameter $\alpha$ is a measure for the effective hybridization between the conduction and $f$ electrons, which has different renormalizations depend-

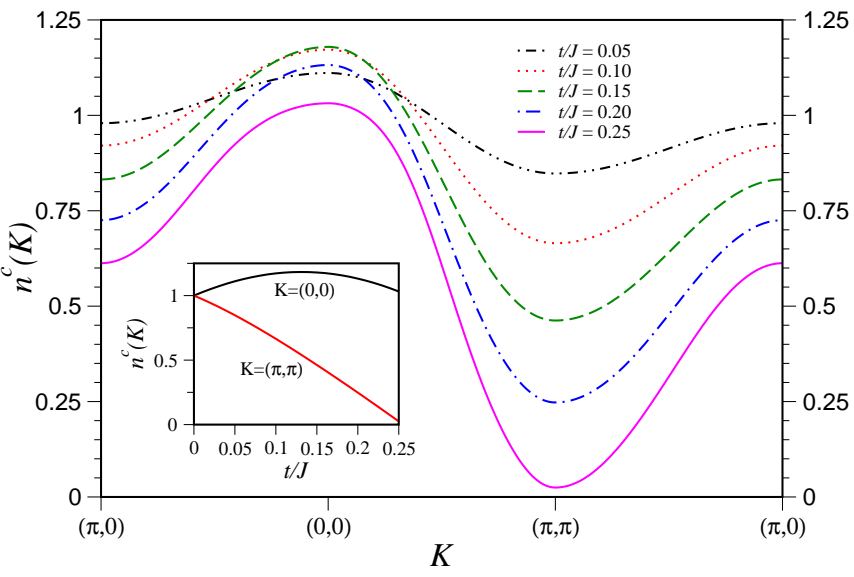

FIG. 10: Integrated weight $n^{c}(K)$ for the two-dimensional Kondo lattice model. The inset shows the particle density at $K=0$ and $K=\pi$ versus the hopping strength.

ing on the corresponding mean field treatment and the model. This form closely resembles our cluster expansion form. We may read out the position of the conduction electron "Fermi surface" defined roughly as the inflection points of $n^{c}(K)$. This yields the point close to $K=\pi / 2$ in one dimension (Fig. 9) and essentially the square-shaped form in two dimensions (Fig. 11). These are the "small" Fermi surfaces which are the relevant ones in the RKKY-picture, where the $f$-electrons are considered as entirely localized spins.

The suppression of $n^{c}(K)$ occurs in the region of the Brillouin zone where the band is getting more and more dispersionless, i.e. this heavy quasiparticle part which may be considered as having "nearly localized $f$-electron" character of the quasiparticles. This aspect looks surprising in view of the fact the Kondo lattice model in a rigorous manner does not allow for $f$-electron charge fluctuations. Thus this behavior is here entirely mediated via the entanglement of conduction and $f$-electron spin. From this viewpoint we may ask when these quasiparticles cease to exist. While our cluster expansion method is definitely unable to give any information beyond the quantum phase transition inside the antiferromagnetic phase, one may still guess on the fate of the heavy quasiparticles. While the antiferromagnetic order will suppress the fluctuations of the localized spins,
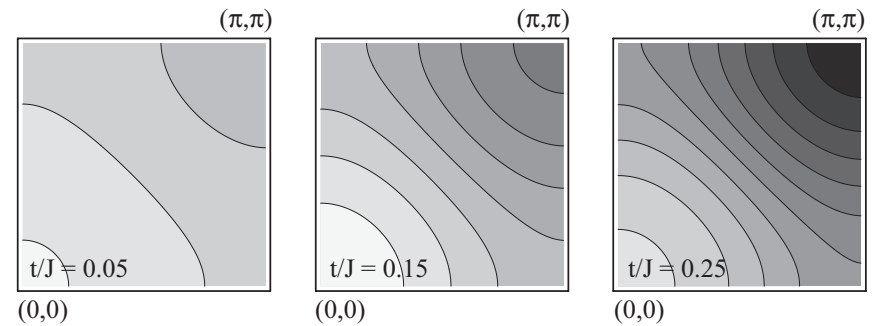

FIG. 11: Contour plot of the integrated weight $n^{c}(K)$ for the two-dimensional Kondo lattice model. 
still there are strong quantum fluctuations leaving space for the entanglement of the $f$ - and conduction electrons. Thus, although it is difficult to show rigorously within our perturbative scheme, we may speculated that the heavy quasiparticles remain in their place even in the magnetically ordered phase for a certain range beyond the quantum phase transition. The heigher weight conduction electron-like quasiparticles together with the shadow band form then gapped quasiparticle band in the reduced Brillouin zone. This would suggest rather a gradual disappearing of the heavy quasiparticles even in the ordered phase.

\section{CONCLUSIONS}

In conclusion, we have studied the dynamics of quasiparticles in the one- and two-dimensional Kondo lattice model at half filling. In the regime of strong Kondo coupling we find typical heavy fermion behavior. The effective quasiparticle mass gets larger as the mobility of the quasiparticle increases and spectral weight is shifted towards the quasiparticle band gap. As the system is driven towards (quasi)long-ranged antiferromagnetic order the shift of quasipartical weigths signals qualitatively the same effects as suggested for the metallic system, the change of the Fermi surface topology. Based on our results we may pose the question whether this change be really abrupt.

Furthermore it is interesting to discuss the effect of the growing spin fluctuation on the two-quasiparticle spectrum. Would there be a tendency towards bound pair formation as we expect from RVB-like systems? Although both the Kondo insulator as well as the RVB system possess short-ranged spin singlet correlations, the Kondo insulator does not show any sign of pair formation. On the opposite, antibound states appear in the two-quasiparticle spectrum.

\section{Acknowledgments}

We thank F. Assaad, I. Milat and H. Tsunetsugu for many helpful discussions. This study has been financially supported by the Swiss National Science Foundation and the NCCR MaNEP.

\section{APPENDIX A: SYMMETRY PROJECTION OF TWO-PARTICLE (ANTI)BOUND STATES}

To compute the two-quasiparticle speactrum we consider the symmetric two-quasiparticle Schrödinger equation 13

\begin{tabular}{c|rrrrr}
$\left(E-E_{0}-E_{1}(K, q)\right)$ & $f(K, q)=$ \\
$C_{4 v}$ & $E$ & $C_{2}$ & $2 C_{4}$ & $2 \sigma_{v}$ & $2 \sigma_{v}^{\prime}$ \\
\hline$A_{1}$ & 1 & 1 & 1 & 1 & 1 \\
$A_{2}$ & 1 & 1 & 1 & -1 & -1 \\
$B_{1}$ & 1 & 1 & -1 & 1 & -1 \\
$B_{2}$ & 1 & 1 & -1 & -1 & 1 \\
$E$ & 2 & -2 & 0 & 0 & 0
\end{tabular}

TABLE I: Characters of irreducible representations of the point group $C_{4 v}$

$$
\frac{1}{N} \sum_{q^{\prime}} f\left(K, q^{\prime}\right)\left(\sum_{a, a^{\prime}} V\left(a, a^{\prime}\right)-E_{1}(K, q)\right)
$$

where $E_{0}$ is the ground state energy, $E_{1}(K, q)$ the combined energy of two scattering quasiparticles and $V\left(a, a^{\prime}\right)$ are the irreducible matrix elements of the calculated twoquasiparticle effective Hamiltonian. The scattering amplitude is denoted as $f(K, q)$. The numerical solution of Eq. (A1) allows to compute the two-quasiparticle continuum and all bound and antibound states.

To determine the symmetry of the antibound state we project the irreducible matrix elements of the effective two-quasiparticle Hamiltonian, $V\left(a, a^{\prime}\right)$, onto the irreducible representations $\gamma$ of the point group $C_{4 v}$

$$
V\left(a, a^{\prime}\right) \longrightarrow F_{a}^{(\gamma)}(q) V\left(a, a^{\prime}\right) F_{a^{\prime}}^{(\gamma)}\left(q^{\prime}\right)
$$

where the projection $F_{a}^{(\gamma)}(q)$ is given by

$$
F_{a}^{(\gamma)}(q)=\frac{1}{\left|C_{4 v}\right|} \sum_{g \in C_{4 v}} \chi_{g}^{(\gamma)} \cos (q \cdot g a)
$$

We subsequently solve the projected two-quasiparticle Schrödinger equation (A1) and thereby identify onto which representation the antibound state can be mapped.
1 S. Doniach, Physica B 91, 231 (1977).

2 P. Coleman, C. Pepin, Q. Si, and R. Ramazashvili, J. Phys.: Condens. Matter 13, R723 (2001).

${ }^{3}$ H. Tsunetsugu, M. Sigrist, and K. Ueda, Reviews of Modern Physics 69, 809 (1997).

${ }^{4}$ Q. Si, S. Rabello, K. Ingersent, and J. L. Smith, Nature 413, 804 (2001).

5 S. Paschen, T. Lühmann, S. Wirth, P. Gegenwart, O. Trovarelli, C. Geibel, F. Steglich, P. Coleman, and Q. Si,
Nature 432, 881 (2004).

${ }^{6}$ F. F. Assaad, Phys. Rev. Lett. 83, 796 (1999).

7 M. Feldbacher, C. Jurecka, F. F. Assaad, and W. Brenig, Phys. Rev. B 66, 045103 (2002).

8 F. F. Assaad, Phys. Rev. B 70, 020402(R) (2004).

${ }^{9}$ K. Beach, P. Lee, and P. Monthoux, Phys. Rev. Lett. 92, 026401 (2004).

10 I. Milat, F. Assaad, and M. Sigrist, Eur. Phys. J. B 38, 571 (2004). 
11 J. R. Schrieffer and P. A. Wolff, Phys. Rev. 149, 491 (1966).

12 M. P. Gelfand and R. R. P. Singh, Advances in Physics 49, 93 (2000).

13 S. Trebst, H. Monien, C. J. Hamer, W. H. Zheng, and R. R. P. Singh, Phys. Rev. Lett. 85, 4373 (2000).

14 W. Zheng, C. Hamer, R. R. P. Singh, S. Trebst, and H. Monien, Phys. Rev. B 63, 144410 (2001).

15 C. Jurecka and W. Brenig, Phys. Rev. B 64, 092406 (2001).

16 N. Shibata and K. Ueda, J. Phys.: Condensed Matter 11, R1 (1999).

17 Z.-P. Shi, R. R. P. Singh, M. P. Gelfand, and Z. Wang, Phys. Rev. B 51, R15630 (1995).

18 W. Zheng and J. Oitmaa, Phys. Rev. B 67, 214406 (2003).
19 T. Rice and K. Ueda, Phys. Rev. Lett. 55, 995 (1985).

20 P. Coleman, Phys. Rev. B 29, 3035 (1984).

21 C. Domb and M. S. Green, Phase Transitions and Critical Phenomena (Academic Press, London, 1974).

${ }^{22}$ C. Knetter, K. P. Schmidt, and G. S. Uhrig, Eur. Phys. J. B 36, 525 (2004).

23 A. M. Tsvelik, Phys. Rev. Lett. 72, 1048 (1994).

24 S. Capponi and F. F. Assaad, Phys. Rev. B 63, 155113 (2001).

${ }^{25}$ K. Ueda, T. Nishino, and H. Tsunetsugu, Phys. Rev. B 50, 612 (1994)

26 N. Read, D. Newns, and S. Doniach, Phys. Rev. B 30, 3841 (1984). 